\title{
LA INVESTIGACIÓN CIENTÍFICA EN HONDURAS DESDE LOS ÁMBITOS ECONÓMICO, EDUCATIVO Y DE SALUD EN EL PERIODO 1980-2015: UNA CARACTERIZACIÓN
}

\author{
Edwin Medina \\ Anayensy Cárcamo Pérez \\ Arely Jackeline Vaquedano Morales \\ Carrera de Lenguas Extranjeras, Facultad de Humanidades y Artes, \\ Universidad Nacional Autónoma de Honduras \\ edwin.medina@unah.edu.hn, anayensy2010@gmail.com, Arely_93@hotmail.es
}

\section{RESUMEN}

Este artículo plantea reunir las tendencias sobre la discusión actual de la investigación científica dentro del sector económico, educativo y salud en Honduras (1980-2015), generando una visión más amplia, no sólo como práctica académica a nivel de universidades e instituciones aisladas o como un simple enfoque teórico o paradigmático, sino como una práctica social en la que los investigadores y las instituciones asuman la investigación científica como un medio de contribuir al desarrollo del país mediante planteamientos propios de gestión del desarrollo, de introducir cambios encaminados a mejorar el sistema y a la aplicación de los conocimientos para transformar la realidad. Además de la caracterización de las investigaciones en estas tres áreas, se cuenta con las valoraciones de varios expertos en cada área, lo que permite un análisis del impacto que esta ha tenido, sus fortalezas y las oportunidades de mejora. Se consideran estos tres ámbitos de investigación por su relación con el desarrollo humano. En este sentido se vuelve imperante conocer y explorar cual es el estado de las investigaciones científicas en Honduras dado que estos son indicadores clave para la construcción y medición de indicadores de Desarrollo Humano.

\section{PALABRAS CLAVE}

Investigación educativa, investigación en salud, investigación económica, desarrollo humano.

\begin{abstract}
This article is an attempt to gather the tendencies about the current discussion in scientific research in the economical, educational and health sectors in Honduras (1980-2015), generating a wider view, not only as an academic practice at universities or isolated institutions or as a simple theoretical or paradigmatical approach but as a social practice in which researchers and institutions adopt scientific research
\end{abstract}

Recibido 19 de agosto de 2016 /Aceptado 10 de noviembre de 2016 
as a means to contribute to the development of the country by means of new ideas to manage development, to bring about change that can improve the system and to the application of knowledge to transform reality.

Besides, the characterization of scientific research in these three areas, the points of view of several experts are included in order to understand the impact that scientific research has had, its strengths and also its opportunities for improvement. These three areas of research are considered given their relation to human development. In this sense, it becomes of utmost importance to know and explore which is the actual state of scientific research in Honduras. The economical aspect is a key element in the development of the countries, in this sense, for the last thirty years the country has been unable to overcome the high poverty and extreme poverty indicators. On the other hand, the low educational level, access, coverage and education quality become obstacles to development. Therefore, educational research becomes fundamental in order to promote and foster human development. As a last element we have the area of health which is related to the permanent search for new knowledge that can provide with new alternatives for decision making and for the creation of public policies in the health area.

\section{KEY WORDS}

Educational research, health research, economic research, human development.

\section{INTRODUCCIÓN}

Los resultados de la investigación muestran que al menos en los últimos 25 años (1980-2015) lo que se ha hecho en Honduras es un análisis DESCRIPTIVO de la realidad del país. Se estudian los mismos temas una y otra vez, se hacen líneas de base, se encuentran los mismos problemas, pero no se plantean alternativas de solución más allá de las “recomendaciones” de los trabajos de investigación. Se entiende claramente que no toda la investigación será utilizada con fines prácticos o que las decisiones de política pública serán tomadas con base en los resultados de las mismas. De acuerdo a lo encontrado, el fin principal de la investigación es académico o ilustrativo (Weiss, 1980) pero por lo general no tiene impacto más allá de ciertos círculos que no son en términos generales los estratos que están sufriendo la problemática. Por otra parte, el hecho de que se “repitan” investigaciones o líneas base está directamente relacionado con la desconexión existente entre los diferentes organismos que realizan investigación y la credibilidad de los datos estadísticos proporcionados por las fuentes oficiales. Esta desconexión nos lleva a duplicar esfuerzos y a resguardar la información en lugar de compartirla. Hay muchas instituciones y organismos tanto gubernamentales como no gubernamentales haciendo investigación, pero al final es una especie de vigores dispersos; todos quieren cubrir todo y solo confían en “sus propios” datos. 
El hecho que la mayoría de la investigación que se realiza en los ámbitos económico, educativo y de salud sea de tipo descriptivo plantea otro problema pues los estudios se realizan de manera atomizada, sin establecer sinergias entre las áreas y sin buscar la complementariedad de los resultados. Contar con estudios descriptivos es válido en una primera fase de análisis de la problemática, sin embargo, se debe continuar escalando en el grado de complejidad de manera que se lleguen a indagar y probar hipótesis de tipo correlacional y explicativo.

Las capacidades instaladas en investigación son como menciona Tunnerman (2000) una muestra del subdesarrollo de nuestros países. La interrogante fundamental que surge ante la problemática de la investigación descriptiva es ¿Se puede con este tipo de investigación contribuir a alcanzar el desarrollo humano? O ¿Aporta este tipo de investigación a la gestión del desarrollo humano? Aunque algunos autores como Licha (2007) plantean que existe una relación directa entre investigación y desarrollo (al menos en los países centrales), cabe preguntarse si la investigación que se realiza en los países subdesarrollados en lugar de "liberarnos” y acercarnos al desarrollo, nos limita y nos mantiene en un círculo vicioso en el que seguimos enfocados en describir los problemas, pero no en buscar alternativas de solución.

\section{MÉTODOS Y MATERIALES}

Poder realizar una caracterización de los procesos de investigación implicó delimitar el área de análisis a algunos aspectos que pudieran ser comunes -comparables- entre las diferentes investigaciones realizadas. En este sentido las características se delimitaron a las siguientes categorías de análisis: Año de publicación, paradigma, enfoque, alcance, temática, autor, metodología e impacto de las investigaciones en las áreas específicas de Salud, Economía y Educación.

Para el desarrollo de la investigación, se planteó un Diseño Hermenéutico que conllevó un análisis comparativo de contenido que se realizó mediante un trabajo bibliográfico accesando a bases de datos especializadas sobre la temática. En el área económica, se revisaron 211 trabajos de investigación, en el área de la salud 380 trabajos y en el área de educación 193, todos realizados y publicados entre 1980 y 2015.

El estudio de las investigaciones realizadas en el país en estas áreas requirió además la realización de entrevistas a informantes clave en cada área; en este sentido se entrevistaron 8 expertos en cada área para un total de 24 especialistas entrevistados. 
El análisis de la información recabada se realizó mediante el modelo planteado por Miles y Huberman (1996) que conlleva una fase de reduccion de datos, una fase de transformacion y una fase de extraccion de conclusiones.

\section{DISCUSIÓN TEÓRICA}

Al hablar de investigación científica y desarrollo existen dos posturas muy bien diferenciadas, por un lado, está la idea de que la investigación científica promueve el desarrollo y por otra parte la idea que es el desarrollo el que promueve la investigación. Desde la primera línea se plantea que existe una relación “innegable” entre investigación y desarrollo (Licha, 2007); en tanto por otra parte se plantea que la investigación científica en los países subdesarrollados es también subdesarrollada y por ende su aporte a la solución de los problemas de la región y al desarrollo social es demasiado limitado. (Herrera, 1995; Tünnerman 2000)

Ahora bien, la investigación científica hace referencia a la aplicación del método científico al estudio de fenómenos sociales como la pobreza y la desigualdad social, como a la generación, adquisición y aplicación de nuevo conocimiento en todos los ámbitos sociales, económicos, políticos y culturales. En términos generales, el fin último de la investigación científica es contribuir al bien común.

La investigación es vista entonces como una vía para llegar al desarrollo (científico, tecnológico, económico, etc.) y por ende cada país genera políticas científicas en pro de la búsqueda de soluciones a los problemas de la región.

En este sentido, Herrera (1995) plantea que el principal problema de la investigación en América latina es precisamente que no cumple con esa misión de búsqueda de soluciones, ya que se encuentra por lo general desconectada de la sociedad a la que pertenecen. En tanto que en los países desarrollados la mayor parte de la investigación se realiza en función de temas relacionados con sus objetivos nacionales.

De ahí que el progreso científico se refleja en su industria, tecnología y en términos generales en un incremento en la producción. La investigación en América Latina por su parte, guarda muy poca relación con los problemas de la región, hay una clara falta de correspondencia entre lo que se investiga y las necesidades de la sociedad. Un factor determinante del atraso (subdesarrollo) investigativo en la región tiene que ver con la pobreza y la escasez de los recursos humanos disponibles. En este sentido se retoma lo que planteaba Harbison (1963) cuando se manifestaba en relación con las capacidades de investigación de los países subdesarrollados y afirmaba que "el problema básico en la mayoría de los 
países subdesarrollados no es la escases de sus recursos naturales, sino el subdesarrollo de sus recursos humanos."

En el mismo sentido incluso la CEPAL (Citado por Herrera, 1995) reconoce esta característica cuando asegura que:

La investigación tecnológica se ha desarrollado como un apéndice de la investigación universitaria de carácter especulativo, como una forma de complementar la enseñanza teórica con el entrenamiento práctico, más que con la intención de atender directamente las necesidades de los medios industriales... (Herrera, 1995, p. 5)

A pesar de las situaciones negativas que enfrenta la investigación científica en los países subdesarrollados, esta también se ha granjeado algunos triunfos que se deben en gran medida a la inyección de la ayuda financiera adecuada por parte del gobierno, el sector privado (mediante la industria) y los organismos internacionales en algunos países. Los éxitos de las economías que han surgido recientemente en el sudeste de Asia (a pesar de la actual crisis económica) también han venido a apoyar la idea de que, en una economía impulsada por la ciencia y la tecnología, la “economía del saber” se vuelve fundamental y está por lo general se desarrolla en las universidades. En la actualidad, es terreno común que los gobiernos esperan que la universidad desempeñe su papel de agente importante del crecimiento económico y el desarrollo. No obstante, ello tiene su precio. La inyección de fondos públicos en la universidad para la investigación exige la rendición de cuentas, que podría depender directamente de un órgano exterior sin recurrir a los procedimientos de evaluación de la universidad. Ello podría influir negativamente en un principio al que la universidad tiene mucha estima, a saber, el respeto de la libertad de cátedra y la libertad de elección a la hora de llevar a cabo la investigación.

Por otra parte, en otros países se plantea el problema de la investigación realizada fuera de la universidad. El gasto público total en investigación se suele repartir entre la universidad y otros institutos o centros de investigación nacionales especializados, mientras que las empresas multinacionales tienen sus propios laboratorios de investigación. La investigación avanzada ha dejado de ser monopolio de la universidad y en términos generales, los resultados obtenidos en institutos nacionales de investigación y en la industria pueden ser confidenciales y no ser revelados al público de la investigación hasta que no han transcurrido dos o tres décadas en algunos casos, por ejemplo, en la investigación bio-médica. Los conocimientos han dejado de difundirse inmediatamente a todos aquéllos que podrían utilizarlos. Es evidente que esta situación complica el principio normativo de la universalidad del conocimiento. De este modo, la libertad de efectuar investigaciones y de difundir sus resultados y la libertad de comunicarse con otros investigadores y de difundir la información relativa a la investigación (estos valiosos derechos) 
están en peligro, lo que acarrea consecuencias que previsiblemente serán nefastas para la formación de la próxima generación de investigadores. (Akyeampong, 1998)

Dado que la ineficiencia o una débil eficiencia de los procesos de investigación tienen un impacto directo sobre los problemas humanos nos parece fundamental acentuar la eficacia de las instituciones y las administraciones de los programas de investigación que puedan utilizar las disponibilidades científicas nacionales y conlleven a la creación de una tradición científica y de un capital de investigación en las sociedades subdesarrolladas. Estos programas de investigación se pueden eventualmente convertir en instrumentos de sus estrategias de desarrollo (Lecerf, 1967) pero para ello es preciso dar centralidad a la cuestión social, y en dicho marco (re)definir la agenda de investigación científica, a través de un proceso democrático que asegure y explicite el compromiso de la ciencia con el desarrollo.

En el caso de nuestro país es también urgente además de pensar la investigación para el desarrollo humano, es decir aquella cuyos resultados promuevan el desarrollo de las capacidades de la gente, se hace necesario pensar en la investigación científica para gestionar el desarrollo humano, es decir una investigación que nos lleve a una comprensión de los procesos de desarrollo pero que además revise los modelos y supuestos teóricos y proponga otras formas de desarrollo.

\section{RESULTADOS}

\section{Investigaciones Económicas}

En cuanto a las investigaciones en el área económica se realizó la búsqueda y evaluación de trabajos y proyectos de investigación realizados desde 1980 hasta la actualidad, con el fin de conocer principalmente la metodología utilizada. A continuación, se presentan los resultados del análisis de estas investigaciones en función de algunos aspectos metodológicos fundamentales y que permiten una visualización del comportamiento de las mismas en los últimos años.

El paradigma de investigación con relación a los estudios económicos analizados, reflejan un uso similar entre el paradigma interpretativo y el positivista, con un uso del $44.5 \%$ para el primero y de

aproximadamente un 47\% para el segundo. Se puede notar una tendencia de investigación desde un paradigma interpretativo en los 80 y la incorporación de investigaciones socio críticas a partir del año 2000. 
Tabla 1 Investigaciones económicas según paradigma y año de publicación

\begin{tabular}{|l|c|c|c|c|c|}
\hline Año de & \multicolumn{4}{c|}{ Paradigma } & \multicolumn{2}{c|}{ Total } \\
\hline Publicación & $\begin{array}{l}\text { Sin } \\
\text { Especificar }\end{array}$ & Interpretativo & Positivista & $\begin{array}{l}\text { Socio } \\
\text { crítico }\end{array}$ \\
\hline $\mathbf{8 0}$ 's & 0 & 8 & 1 & 0 & 9 \\
\hline $\mathbf{9 0}$ 's & 0 & 6 & 10 & 0 & 16 \\
\hline $\mathbf{2 0 0 0 - 2 0 0 9}$ & 0 & 40 & 40 & 10 & 90 \\
\hline $\mathbf{2 0 1 0}$ a la & 1 & 40 & 48 & 7 & 96 \\
\hline actualidad & & & & & \\
\hline Totales & $\mathbf{1}$ & $\mathbf{9 4}$ & $\mathbf{9 9}$ & $\mathbf{1 7}$ & $\mathbf{2 1 1}$ \\
\hline
\end{tabular}

Asimismo, se observa que existe un predominio de investigaciones económicas bajo un enfoque cuantitativo, alrededor del 56\% de estas siguen esa línea. Es importante destacar que contrario a lo que podría pensarse existe una amplia cantidad de investigaciones bajo la óptica cualitativa con un 39\%. Se observa también que las tendencias favorecen a los estudios cuantitativos con el pasar de los años.

Tabla 2 Investigaciones económicas según enfoque y año de publicación

\begin{tabular}{|l|c|c|c|c|c|}
\hline Año de & \multicolumn{5}{c}{ Enfoque } \\
\hline Publicación & Sin & Cualitativo & Cuantitativo & Mixto \\
\hline & Especificar & & & & \\
\hline $\mathbf{8 0}$ 's & 0 & 7 & 2 & 0 & 9 \\
\hline $\mathbf{9 0}$ 's & 0 & 5 & 10 & 1 & 16 \\
\hline $\mathbf{2 0 0 0 - 2 0 0 9}$ & 2 & 38 & 46 & 4 & 90 \\
\hline $\mathbf{2 0 1 0}$ a la & 1 & 32 & 60 & 3 & 96 \\
\hline actualidad & & & & & \\
\hline Totales & $\mathbf{3}$ & $\mathbf{8 2}$ & $\mathbf{1 1 8}$ & $\mathbf{8}$ & $\mathbf{2 1 1}$ \\
\hline
\end{tabular}

Durante el análisis se encontró que alrededor del 36 por ciento de los estudios realizados en el área económica son descriptivos, seguidos por un 23 por ciento que son correlacionales y un 20\% que son 
explicativas. Las investigaciones de carácter documental ocupan un 15\% de las investigaciones. A partir del año 2000 se suman las investigaciones exploratorias y los estudios de caso.

Tabla 3 Investigaciones económicas según alcance y año de publicación

\begin{tabular}{|c|c|c|c|c|c|}
\hline \multirow[t]{2}{*}{ Alcance } & \multicolumn{4}{|c|}{ Año de Publicación } & \multirow[t]{2}{*}{ Total } \\
\hline & $80^{\prime} \mathrm{s}$ & 90 's & $2000-2009$ & $\begin{array}{l}2010 \text { a la } \\
\text { actualidad }\end{array}$ & \\
\hline Sin especificar & 0 & 0 & 0 & 1 & 1 \\
\hline Exploratoria & 1 & 1 & 2 & 1 & 5 \\
\hline Descriptiva & 2 & 4 & 31 & 36 & 73 \\
\hline Correlacional & 0 & 4 & 25 & 19 & 48 \\
\hline Explicativa & 1 & 4 & 20 & 18 & 43 \\
\hline Documental & 5 & 3 & 5 & 19 & 32 \\
\hline Histórica & 0 & 0 & 1 & 0 & 1 \\
\hline Estudio de Caso & 0 & 0 & 6 & 2 & 8 \\
\hline Totales & 9 & 16 & 90 & 96 & 211 \\
\hline
\end{tabular}

El diseño no experimental es el más utilizado en las investigaciones analizadas, dado que en su conjunto representan el 76\% de los mismos, el uso de este es predominante a lo largo de los años. Esto muestra que la mayoría de investigación está enfocada desde una visión positivista, pero no llega a plantear modelos experimentales, es decir la investigación sigue describiendo y en algunos casos buscando relaciones, pero no se hacen pruebas (experimentos) que permitan la comprobación de hipótesis

\section{Investigaciones Educativas}

Los Autores y organismos que promueven la investigación educativa en el país se distribuye de la siguiente manera: Academia 95\%, sector privado 2\%, ONG 1\% y sector público 1\%; en lo cual destaca que la academia es el sector que más investigaciones en el área educativa produce en el país. (Y en términos generales en todas las áreas) Para detallar de una mejor manera la identificación de las instituciones pertenecientes a cada sector, a continuación, se presenta los datos en la tabla siguiente. 
Tabla 4 Investigaciones educativas en Honduras 1980-2015 según institución

\begin{tabular}{|l|c|c|}
\hline Institución & $\begin{array}{c}\text { Cantidad de } \\
\text { investigaciones }\end{array}$ & $\begin{array}{c}\text { Porcentaje de } \\
\text { investigaciones }\end{array}$ \\
\hline UNAH & 43 & $22 \%$ \\
\hline $\begin{array}{l}\text { Organismos de } \\
\text { Cooperación }\end{array}$ & 5 & $3 \%$ \\
\hline UNESCO & 1 & $1 \%$ \\
\hline UNICAH & 5 & $3 \%$ \\
\hline UNION EUROPEA & 1 & $1 \%$ \\
\hline UNITEC & 6 & $3 \%$ \\
\hline $\begin{array}{l}\text { Universidades } \\
\text { internacionales }\end{array}$ & 4 & $2 \%$ \\
\hline UPNFM & 123 & $64 \%$ \\
\hline No identificado & 5 & $3 \%$ \\
\hline Total & 193 & $100 \%$ \\
\hline
\end{tabular}

Las investigaciones realizadas se encontraron en diferentes formatos, $63 \%$ son tesis de maestría, $30 \%$ son artículos en revistas científicas, $4 \%$ son informes técnicos y 3\% son tesis doctorales. Siguiendo la línea del análisis anterior, siendo las tesis el formato de informe que predomina, en éste tienen un fuerte peso las tesis de maestría de la UPNFM. El rango de aplicación de las investigaciones pertenece 37\% a educación superior, 30\% a educación media, 20\% a educación básica, 11\% una aplicación transversal, es decir, a la vez en varios sectores y 1\% a educación pre básica.

Tabla 5 Investigaciones educativas en Honduras 1980-2015 según período

\begin{tabular}{|l|r|r|}
\hline Período & $\begin{array}{l}\text { Cantidad de } \\
\text { investigaciones }\end{array}$ & $\begin{array}{l}\text { Porcentaje de } \\
\text { investigaciones }\end{array}$ \\
\hline $\mathbf{8 0 s}$ & 1 & $0.5 \%$ \\
\hline $\mathbf{9 0 s}$ & 1 & $0.5 \%$ \\
\hline $\mathbf{2 0 0 0 - 2 0 0 9}$ & 86 & $44 \%$ \\
\hline $\mathbf{2 0 1 0}$ a la \\
actualidad
\end{tabular}


El período con más investigaciones educativas realizadas es el 2000 a la actualidad, lo que está directamente relacionado con el aumento de las tesis de maestría de la UPNFM y también con el aumento de la oferta de posgrados en dicha universidad.

En cuanto a la metodología empleada para la realización de las investigaciones en el ámbito educativo, las investigaciones analizadas presentan una diversidad de diseños metodológicos, estos se describen detalladamente a continuación. Sobre los paradigmas, el positivista fue utilizado en el 47\% de las investigaciones y el interpretativo en el 25\%; el 28\% de las investigaciones no explicitan el paradigma utilizado.

En cuanto al enfoque, el más utilizado fue el cualitativo, que está presente en el $41 \%$ de las investigaciones, seguido por el cuantitativo en el 39\% y por último el mixto en el $18 \%$ de las investigaciones; cabe mencionar que el $1 \%$ de las investigaciones no tenía explícito este dato. Para detallar más en los aspectos metodológicos, la siguiente tabla muestra los diseños empleados en las investigaciones educativas.

Tabla 6 Investigaciones educativas en Honduras 1980-2015 según diseño de investigación

\begin{tabular}{|c|c|c|}
\hline Diseño & $\begin{array}{l}\text { Cantidad de } \\
\text { investigaciones }\end{array}$ & $\begin{array}{l}\text { Porcentaje de } \\
\text { investigaciones }\end{array}$ \\
\hline Experimental & 14 & $7 \%$ \\
\hline No experimental & 107 & $55 \%$ \\
\hline Fenomenológico & 20 & $10 \%$ \\
\hline Etnográfico & 7 & $4 \%$ \\
\hline $\begin{array}{l}\text { Investigación } \\
\text { acción }\end{array}$ & 15 & $8 \%$ \\
\hline $\begin{array}{l}\text { Teoría } \\
\text { fundamentada }\end{array}$ & 4 & $2 \%$ \\
\hline Narrativo & 5 & $3 \%$ \\
\hline Documental & 14 & $7 \%$ \\
\hline No identificado & 7 & $4 \%$ \\
\hline Total & 193 & $100 \%$ \\
\hline
\end{tabular}


En los diseños, el más utilizado, al igual que en las investigaciones económicas, fue el No experimental, con una cantidad bastante superior a los demás diseños, los cuales se mantienen en porcentajes similares. Sobre los alcances utilizados, destaca como el primero el descriptivo con el $49 \%$ de las investigaciones, seguido por el correlacional con 19\%, después el exploratorio con 15\% y por último el explicativo con 12\% porcentaje aún más bajo que el de las investigaciones en el área económica; cabe mencionar que en el $5 \%$ de las investigaciones, no se especifica el alcance.

En cuanto al impacto o aporte que se logra a través de estas investigaciones, se encontraron dos tipos de aportes más utilizados siguiendo la taxonomía de Carol Weiss (1980), uno es el de resolución de problemas, que implica, a través del conocimiento generado, resolver un problema específico del tomador de decisiones. Este modelo implica que hay acuerdo o consenso general entre los investigadores y los hacedores de política en la solución o estado final del problema. El otro tipo de aporte es el iluminado, en el cual la investigación gradualmente informa sobre los problemas y la identificación de opciones de política. Sirve para “educar” al hacedor de política, es decir, con el tiempo la acumulación de investigación influirá en la política, luego de que ha estado instruyendo al hacedor de política. (Weiss, 1980)

Tabla 71 Investigaciones educativas Honduras 1980-2015 según tipo de aporte por el modelo Weiss

\begin{tabular}{|l|c|c|}
\hline Tipo de aporte & $\begin{array}{c}\text { Cantidad de } \\
\text { investigaciones }\end{array}$ & $\begin{array}{c}\text { Porcentaje de } \\
\text { investigaciones }\end{array}$ \\
\hline Iluminado & 54 & $28 \%$ \\
\hline Interactivo & 38 & $20 \%$ \\
\hline Lineal & 33 & $17 \%$ \\
\hline Político & 10 & $5 \%$ \\
\hline Resolución de & 56 & $29 \%$ \\
\hline problemas & & \\
\hline Táctico & 1 & $1 \%$ \\
\hline No identificado & 1 & $1 \%$ \\
\hline Total & 193 & $100 \%$ \\
\hline
\end{tabular}




\section{Investigaciones en Salud}

En Honduras la investigación en salud ha dado pasos significativos. El sistema de salud hondureño está constituido por el sector público, compuesto por la Secretaría de Salud y el IHSS, y el sector no público o privado, con y sin fines de lucro. En términos de investigación los trabajos científicos que se realizan son liderados por la Universidad Nacional Autónoma de Honduras (UNAH).

En el área de salud la mayor cantidad de investigaciones se realizaban en los años 80’s desde un paradigma positivista, lo cual es acorde a su tradición de formación como parte de las ciencias “duras”. A partir de los años 90, sin embargo se ha venido dando un incremento de investigaciones que se realizan desde un paradigma interpretativo; es decir investigaciones que más que explicar y predecir los fenómenos como se hace desde el positivismo, busca profundizar en las causas de los fenómenos.

\section{Tabla 82 Investigaciones en salud según enfoque}

\begin{tabular}{|l|c|c|c|c|c|}
\hline Año de & \multicolumn{5}{|c|}{ Enfoque } \\
\hline & $\begin{array}{l}\text { Sin } \\
\text { Publicación }\end{array}$ & Cualitativo & Cuantitativo & Mixto \\
\hline $\mathbf{8 0}$ Es $^{\prime}$ & 0 & 17 & 56 & 0 & 73 \\
\hline $\mathbf{9 0}$ 's & 0 & 65 & 10 & 0 & 75 \\
\hline $\mathbf{2 0 0 0 - 2 0 0 9}$ & 1 & 63 & 5 & 0 & 69 \\
\hline $\mathbf{2 0 1 0}$ a la & 1 & 43 & 58 & 0 & 102 \\
\hline actualidad & & & & & \\
\hline Totales & $\mathbf{2}$ & $\mathbf{1 8 8}$ & $\mathbf{1 2 9}$ & $\mathbf{0}$ & $\mathbf{3 1 9}$ \\
\hline
\end{tabular}

Acorde con los datos anteriores sobre el paradigma, el enfoque de investigación empleado a partir de los años 90 es el cualitativo. Esto implica que se han enfatizado los estudios en el área de la salud en conocer aspectos cualitativos de la problemática en salud, sin embargo, esto plantea el reto de que la investigación cualitativa por lo general no busca probar hipótesis o teorías.

Otro aspecto interesante en las investigaciones realizadas en el área de salud es que estas han sido realizadas ya sea por la academia (133) y la mayoría por la sociedad civil. Esto parece indicar que por parte del gobierno y el sector privado, el sector salud no es una de sus prioridades en investigación. 
Tabla 9 Investigaciones en salud según organismo

\begin{tabular}{|l|r|r|r|r|r|}
\hline \multicolumn{1}{|c|}{ Organismo Año de Publicación } \\
\hline & 80`s & 90`s & $2000-2009$ & $\begin{array}{l}\text { 2010 a la } \\
\text { actualidad }\end{array}$ \\
\hline Academia & 22 & 88 & 10 & 13 & 133 \\
\hline Independientes & 0 & 0 & 0 & 5 & 5 \\
\hline Sector Privado & 0 & 0 & 0 & 0 & 0 \\
\hline Sector Publico & 0 & 0 & 0 & 0 & 0 \\
\hline Sociedad Civil & 55 & 73 & 106 & 102 & 336 \\
\hline Totales & 77 & 161 & 116 & 120 & 474 \\
\hline
\end{tabular}

Como punto final es importante destacar la relevancia que posee la investigación como tal, dado que es considerada el punto de partida para generar incidencia y cambios razonados, que vayan acorde a las políticas de país, aunque es lamentable que se vean opacadas por ideales políticos o por no proceder de organismos internacionales, por tal motivo no todas las investigaciones o proyectos logran desembocar en una política pública; quedando limitados los aportes de estas investigaciones que independientemente de su calidad no logran figurar e incentivar el cambio y desarrollo de Honduras.

\section{CONCLUSIONES}

La mayoría de investigación está enfocada desde una visión positivista, pero no llega a plantear modelos experimentales, es decir la investigación sigue describiendo y en algunos casos buscando relaciones, pero no se hacen pruebas (experimentos) que permitan la comprobación de hipótesis. No se ha fortalecido la integración metodológica, por lo general la investigación o es cuantitativa o cualitativa.

Actualmente se investiga predominantemente temas del área macroeconómica, manteniéndose la herencia investigativa en este campo, dentro de la misma cabe destacar que en su mayoría la investigación desde el punto de vista epistemológico es cuantitativa y siguiendo esta línea se observa que los estudios son predominantemente sobre investigación aplicada de tipo descriptivo. 
El mayor aporte de investigaciones educativas lo tienen las maestrías de la UPNFM, por eso es que en los registros el tipo de documento con más frecuencia son las tesis de maestría, las cuales se centran en educación media.

Las investigaciones en el área de la salud se han obtenido hallazgos importantes que provocan discusiones con significativos aportes al conocimiento y a la academia, pero estos no están alcanzando sobrepasar la barrera de la discusión, es decir no están llegando como insumo fundamental a los tomadores de decisión o los hacedores de política en salud.

Una de las principales debilidades encontradas es a nivel metodológico, pues se detecta incoherencia entre paradigmas, enfoques y métodos (diseños) de investigación. Se detectaron además problemas con el manejo del muestreo, lo que puede llevar a la invalidez de los datos presentados por debilidades metodológicas y por ende, a perder datos y recursos valiosos por debilidades metodológicas o técnicas.

\section{RECOMENDACIONES}

Se recomienda continuar fortaleciendo la enseñanza-aprendizaje de la investigación particularmente a nivel de pregrado, de manera que los estudiantes puedan contar con bases sólidas de investigación y al estudiar un posgrado puedan plantearse investigaciones metodológicamente más sólidas y relevantes.

Pasar de lo meramente descriptivo a lo correlacional y experimental es un proceso que pasa por la formación antes mencionada, pero además implica la utilización de la información y resultados ya obtenidos, de manera que los esfuerzos anteriores sean potenciados y utilizados de manera sistemática.

El desafío para la investigación científica en el país es fomentarla como herramienta para la toma de decisiones, el investigador debe de comprender que la investigación es parte de su actividad cotidiana, pero no solo el fomento de la investigación también hay que potencializarla formando alianzas estratégicas instituciones del gobierno, academia e instituciones privadas con miras a fortalecer la investigación desde los recursos económicos, formación del recurso humano que sean capaces de realizar investigaciones sólidas sobre temas relevantes que esté orientada a resolver problemas. 


\section{REFERENCIAS BIBLIOGRÁFICAS}

Akyeampong, D. (1998). La educacion Superior y la investigacion: Desafios y oportunidades. Ghana: ICSU.

Hernández, R., Fernández, C., \& Baptista, P. (2010). Metodologia de la Investigacion 5ta Edicion. Mexico: MacGraw Hill.

Herrera, A. (1995). Los determinantes sociales d ela politica cientifica en America ALtina. Politica cientifica explicita y politica cientifica implicita. REDES, 34-63.

Lecerf, D. (1967). Lénfondrement du systheme. Paris: Synthese.

Licha, I. (2007). Investigacion cientifica y desarrollo social en America Latina. Mexico: UDUAL.

Miles, M., \& Huberman, M. (1996). Qualitative data analysis: An extended Sourcebook. Washington: McGraw Hill.

Tunnerman, C. (2000). Universidad y Sociedad: Balance hostorico y perspectivas desde Latinoamerica. Caracas: UCV.

Weiss, C. (1980). Social Science research and decision-making. New York: Columbia University Press. 